OPEN ACCESS

Edited by:

Manuel Kleiner,

University of Calgary, Canada

Reviewed by:

Guang Zhao,

Chinese Academy of Sciences, China

Dmitri Mavrodi,

The University of Southern

Mississippi, USA

Lars E. P. Dietrich,

Columbia University, USA

*Correspondence:

Mariadhas Valan Arasu

mvalanarasu@gmail.com;

Sang Un Park

supark@cnu.ac.kr

†These authors have contributed equally to this work.

Specialty section: This article was submitted to Microbial Physiology and Metabolism,

a section of the journal

Frontiers in Microbiology

Received: 01 September 2015

Accepted: 29 February 2016

Published: 14 March 2016

Citation:

Thwe A, Valan Arasu M, LiX,

Park CH, Kim SJ, Al-Dhabi NA and Park SU (2016) Effect of Different Agrobacterium rhizogenes Strains on

Hairy Root Induction and Phenylpropanoid Biosynthesis in Tartary Buckwheat (Fagopyrum tataricum Gaertn).

Front. Microbiol. 7:318. doi: 10.3389/fmicb.2016.00318

\section{Effect of Different Agrobacterium rhizogenes Strains on Hairy Root Induction and Phenylpropanoid Biosynthesis in Tartary Buckwheat (Fagopyrum tataricum Gaertn)}

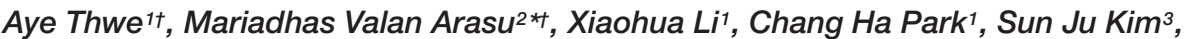
Naif Abdullah Al-Dhabi ${ }^{2}$ and Sang Un Park ${ }^{1 *}$

'Department of Crop Science, Chungnam National University, Daejeon, South Korea, ${ }^{2}$ Department of Botany and Microbiology, Addiriyah Chair for Environmental Studies, College of Science, King Saud University, Riyadh, Saudi Arabia, ${ }^{3}$ Department of Bio-Environmental Chemistry, Chungnam National University, Daejeon , South Korea

The development of an efficient protocol for successful hairy root induction by Agrobacterium rhizogenes is the key step toward an in vitro culturing method for the mass production of secondary metabolites. The selection of an effective Agrobacterium strain for the production of hairy roots is highly plant species dependent and must be determined empirically. Therefore, our goal was to investigate the transformation efficiency of different $A$. rhizogenes strains for the induction of transgenic hairy roots in Fagopyrum tataricum 'Hokkai T10' cultivar; to determine the expression levels of the polypropanoid biosynthetic pathway genes, such as ftpAL, FtC4H, Ft4CL, FrCHS, FrCH1, FrF3H, FtFLS1, FtFLS2, FtF3, H1, FtF3'H2, FtANS, and FtDFR; and to quantify the in vitro synthesis of phenolic compounds and anthocyanins. Among different strains, R1000 was the most promising candidate for hairy root stimulation because it induced the highest growth rate, root number, root length, transformation efficiency, and total anthocyanin and rutin content. The R1000, 15834, and A4 strains provided higher transcript levels for most metabolic pathway genes for the synthesis of rutin (22.31, 15.48, and $13.04 \mu \mathrm{g} / \mathrm{mg}$ DW, respectively), cyanidin 3-O-glucoside (800, 750, and $650 \mu \mathrm{g} / \mathrm{g}$ DW, respectively), and cyanidin 3-O-rutinoside (2410, 1530, and $1170 \mu \mathrm{g} / \mathrm{g}$ DW, respectively). A suitable $A$. rhizogenes strain could play a vital role in the fast growth of the bulk amount of hairy roots and secondary metabolites. Overall, R1000 was the most promising strain for hairy root induction in buckwheat.

Keywords: tartary buckwheat, Agrobacterium rhizogenes, polypropanoid biosynthetic genes, rutin, anthocyanin, HPLC

\section{INTRODUCTION}

Fagopyrum tataricum (Gaertn) is commonly known as tartary buckwheat. It belongs to the Polygonaceae family and is mainly cultivated in the mountain regions of China, Korea, Japan, India, USA, Europe, Brazil, Canada, Australia, and South Africa. F. tataricum is known as a functional food (due to the presence of proteins and amino acids) and as a traditional medicinal 
plant (due to the presence of phenolic compounds). Tartary buckwheat has a bitter taste. Therefore, its consumption has decreased. However, the plant has received increased attention due to the presence of significant amounts of pharmacologically important phenolic compounds, such as quercetin-3-glycoside, kaempferol-3-glycoside, chlorogenic acid, iso-orientin, orientin, rutin, vitexin, and quercitrin (Lee et al., 2014). In addition, it contains dietary fibers, proteins, starch, polyunsaturated fatty acids, and vitamin B and C complexes (Bonafaccia et al., 2003). Phenolic compounds, such as rutin and anthocyanins, that have been identified in buckwheat showed better in vitro antioxidant activity and in vitro pharmacological functions, such as cholesterol reduction, tumor inhibition, anti-hypertension, and control of diabetes and carcinogenesis (Kayashita et al., 1997; Chan, 2003; Kawa et al., 2003; Ishii et al., 2008).

Various strategies have been demonstrated for the synthesis and production of pharmaceutically important phytochemicals (Nagella et al., 2013). These strategies include Agrobacteriummediated gene transfer, in vitro cell line establishment, in vitro cell suspension cultures, bioreactor cultivation, shoot cultivation, organ cultivation at the bioreactor level, and in vitro hairy root cultivation (Bourgaud et al., 2001). However, among these techniques, Agrobacterium-mediated gene transfer has been widely studied as a strategy for producing hairy root lines with high yield for plant compounds (Ali et al., 2008). Agrobacterium is a Gram-negative soil-borne bacterium that is able to transfer part of its DNA (i.e., T-DNA carried on a large plasmid) to a host plant cell (Tzfira et al., 2004). The hairy roots obtained by infecting plants with Agrobacterium rhizogenes are unique with respect to their genetic and biosynthetic stability and have been intensively used to induce a stable and high yield production of selected plant metabolites. A. rhizogenes are appropriate for the production of valuable secondary metabolites, because they can enhance growth regulators and are characterized by fast growth (Shen et al., 1988; Wysokinska and Chmiel, 1997; Giri and Narasu, 2000). Currently, modern molecular techniques are used to regulate metabolic pathway genes that are required for the production of secondary metabolites (Georgiev et al., 2012; Sharma et al., 2013). Additionally, hairy roots are considered as a potential source of new natural compounds. Li et al. (1998) obtained a novel antimicrobial compound, licoagrodione, from Glycyrrhiza glabra using a hairy root culture, and a new tropane alkaloid ester was obtained from Datura stramonium using a hairy root culture (Berkov et al., 2003). Furthermore, growth regulators are not required for the hairy root cultivation, which is an important consideration because some plant hormones are toxic (e.g., 2,4-dichlorophenoxyacetic acid; Shanks and Morgan, 1999). Therefore, hairy root culture provides possibilities for isolating and synthesizing new compounds with high pharmaceutical properties (Kwon et al., 1997).

Many strains of $A$. rhizogenes exist and have been used for plant transformation. Recently, Ooi et al. (2013) and Sudha et al. (2013) used A. rhizogenes to regulate genes that were involved in the plant secondary metabolite production. The selection of an effective Agrobacterium strain for the production of transformed root cultures significantly depends on the plant species and must be determined empirically. The differences in virulence, morphology, and growth rate are at least partially related to the variety of Ri (root inducing)-plasmids within each bacterial strain (Park and Facchini, 2000). Among the T-DNA genes in Ri-plasmid, the rol oncogenes cause striking phenotypical and biochemical alterations in the transformed hairy root. The rol genes are potential activators of the secondary metabolism in transformed cells from the Solanaceae, Araliaceae, Rubiaceae, Vitaceae, and Rosaceae families (Bulgakov et al., 2008). It is largely unknown whether different $A$. rhizogenes strains vary in their abilities to stimulate secondary metabolite production.

In our previous study, we claimed that $F$. tataricum infected with $A$. rhizogenes produced significant amounts of phenylpropanoid (Thwe et al., 2013) (Figure 1). However, to date, there are no reports on the transformation of F. tataricum via different $A$. rhizogenes strains for the phenylpropanoid production. Therefore, in the present study, we aimed to develop a novel hairy root culture method for the production of pharmacologically important phenolic compounds. Different A. rhizogenes strains were screened for the infection, and the expression levels of phenylpropanoid biosynthetic genes were evaluated.

\section{MATERIALS AND METHODS}

\section{Chemicals and Reagents}

HPLC-grade methanol, acetonitrile, and formic acid were obtained from Wako Pure Chemical Industries (Osaka, Japan). Phenolic compound standards for rutin, catechin, chlorogenic acid, benzoic acid, ferulic acid, and quercetin were procured from Extrasynthese (Genay, France). Cyanidin 3-O-rutinoside and cyanidin-3-O-glucoside were purchased from Fujicco Co., Ltd. (Kobe, Japan). Other routine reagents and chemicals were procured from Sigma-Aldrich (St. Louis, MO, USA).

\section{Plant Materials}

Tartary buckwheat ('Hokkai T 10') seeds were kindly gifted by the Hokkaido Agricultural Research Center, Sapporo, Japan, in July 2011. For the hairy root stimulation, the seeds were disinfected with $70 \%(\mathrm{v} / \mathrm{v})$ ethanol for $120 \mathrm{~s}$, followed by rinsing with $4 \%$ (v/v) sodium hypochlorite solution $(\mathrm{NaClO})$ for $15-20 \mathrm{~min}$ and with Tween-20 for $15 \mathrm{~min}$. The seeds were mixed with icecold autoclaved sterile distilled water three to five times and completely washed five times to remove any trace elements. The surface-sterilized seeds were wiped with sterile cotton to remove water droplets and, then the seeds were transferred into a sterile culture flask containing $25 \mathrm{ml}$ of autoclaved half-strength MS (Murashige and Skoog, 1962) without hormones. The seeds were incubated at $25^{\circ} \mathrm{C}$ under light with an intensity of $35 \mu \mathrm{mol} \mathrm{s}{ }^{-1}$ $\mathrm{m}^{-2}$ for 3 days to facilitate seed germination. The sterile bottle with seeds was maintained in a Magenta box containing $50 \mathrm{ml}$ of the same basal medium under 16/8-h light/dark conditions for the growth of seedlings at $25 \pm 1^{\circ} \mathrm{C}$.

\section{Bacterial Strains and Maintenance}

Wild-type A. rhizogenes (R1000), R1200, R13333, R15834, R1601, LBA9402, and A4 were used to determine the 


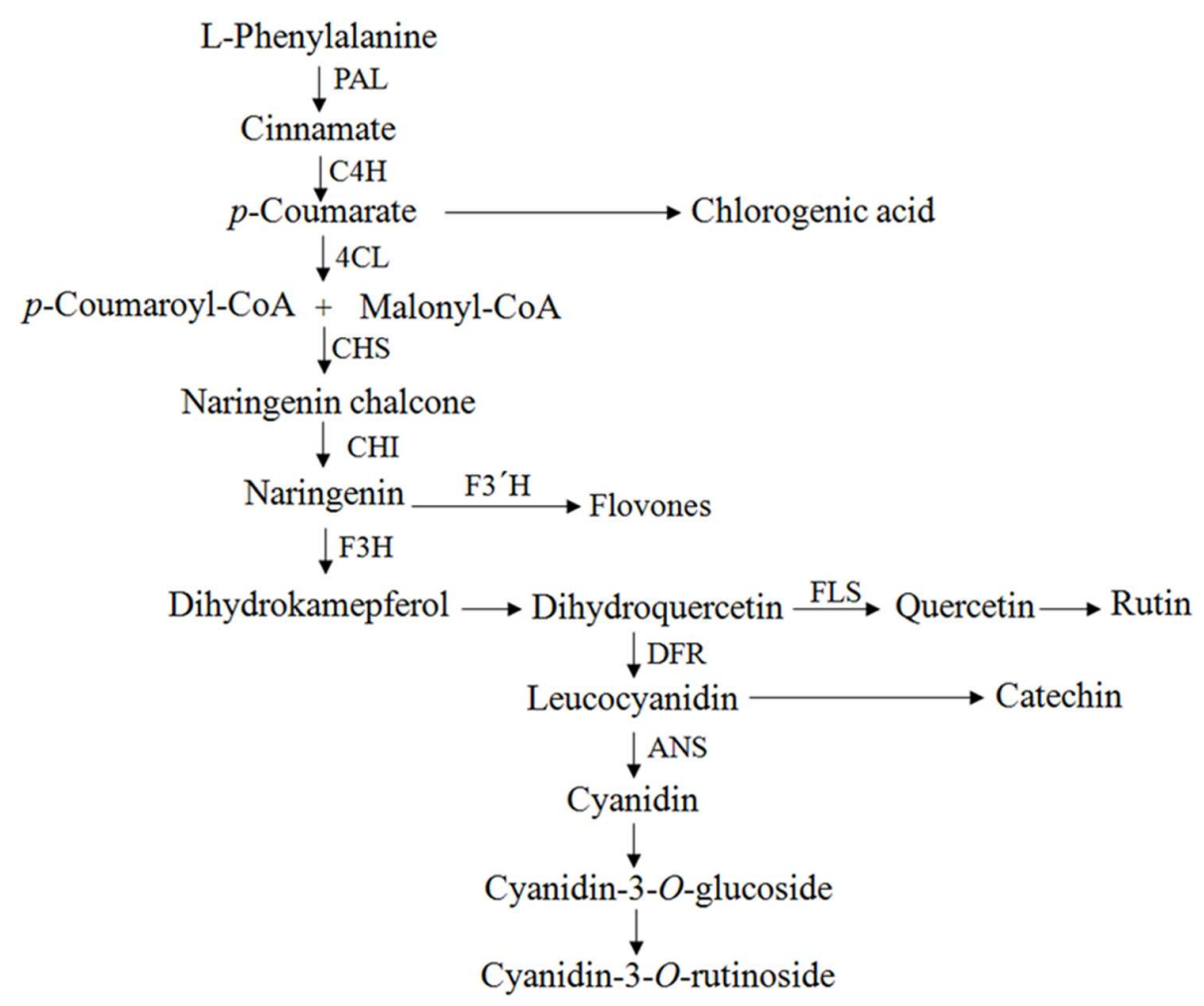

FIGURE 1 | Flavonoid pathway scheme. PAL, phenylalanine ammonia lyase; C4H, cinnamate 4-hydroxylase; 4CL, 4-coumaroyl CoA ligase; CHS, chalcone synthase; CHI, chalcone isomerase; F3H, flavones 3-hydroxylase; FLS, flavonol synthase; F3'H, flavonoid 3'-hydroxylase; ANS, anthocyanidin synthase; DFR, dihydroflavonol-4 reductase.

transformation efficiency. The strains were kindly gifted by Centro de Investigacion Cientifica de Yucatan, Mexico, and were maintained in our laboratory. The bacterial strains were cultivated in an LB medium containing $1 \mathrm{~g}$ of tryptone, $0.5 \mathrm{~g}$ of yeast extract, and $0.5 \mathrm{~g}$ of $\mathrm{NaCl}$ in a $100-\mathrm{ml}$ flask at $28^{\circ} \mathrm{C}$ and $224 \mathrm{rcf}$ for $24 \mathrm{~h}$. All of the strains were maintained in $20 \%$ glycerol at $-80^{\circ} \mathrm{C}$. Viability of the strains was determined intermittently by growing in LB agar.

\section{Hairy Root Induction}

Hairy root induction was performed using a slightly modified method of Thwe et al. (2013). Briefly, $5 \mathrm{ml}$ of freshly cultivated $A$. rhizogenes strains at the mid-log phase $\left(\mathrm{A}_{600}=0.6\right)$ were centrifuged at $5600 \mathrm{rcf}$ for $10 \mathrm{~min}$. Cell pellets were further washed with an ice-cold phosphate buffer ( $\mathrm{pH} 7$ ) and resuspended in half-strength MS liquid medium. The explants were dipped into suspensions of different $A$. rhizogenes strains for $20 \mathrm{~min}$, dried on a sterile tissue paper in a sterile bottle, and incubated under dark conditions at $25 \pm 1^{\circ} \mathrm{C}$ on a half-strength solid MS medium for 2 days. After incubation, the explants were carefully collected and rinsed with sterile distilled water and cultivated in a hormone-free half-strength MS medium with $500 \mathrm{mg} / \mathrm{l}$ of cefotaxime. After 7 days of incubation, the emerged hairy roots were further sub-cultured in a flask containing a halfstrength MS medium for 21 days at $25 \pm 1{ }^{\circ} \mathrm{C}$. After incubation, the hairy roots were harvested to examine the gene expression levels and to quantify the phenolic compounds. The collected hairy roots were carefully stored in a clean container at $-80^{\circ} \mathrm{C}$ for further chemical and molecular analyses.

\section{Extraction of Genomic DNA and PCR Analysis}

Genomic DNA of transformed tartary buckwheat was extracted using a DNeasy Plant Mini Kit (Qiagen; Valencia, CA, USA). The primers for the fragments of $r o l$ genes ( $r o l \mathrm{~A}, \mathrm{~B}, \mathrm{C}$, and D) are listed in Table 1. The conditions for thermal amplification of the rol genes were as follows: initial denaturation at $95^{\circ} \mathrm{C}$ for $2 \mathrm{~min}$, followed by 30 cycles of amplification at $95^{\circ} \mathrm{C}$ for $30 \mathrm{~s}$, primer annealing at $55^{\circ} \mathrm{C}$ for $45 \mathrm{~s}$, and primer extension at $72^{\circ} \mathrm{C}$ for $1 \mathrm{~min}$ and final extension at $10 \mathrm{~min}$ and cooling to $4^{\circ} \mathrm{C}$. The purity of amplified products was checked by mixing $10 \mu \mathrm{l}$ of the PCR products with a loading dye and by performing electrophoresis on $1 \%$ agarose gels that were prepared in a $0.5^{\star}$ TBE (Tris/Borate/EDTA) buffer. The gels were analyzed for fragment sizes of the $\operatorname{rol}$ genes $(\operatorname{rol} \mathrm{A}, \mathrm{B}, \mathrm{C}$, and D).

\section{Extraction of the Total RNA and cDNA Synthesis}

The RNeasy Plant Mini Kit (Qiagen; Valencia, CA, USA) was used to extract the total RNA from the wild and transformed tartary buckwheat hairy roots. The purity of RNA was evaluated 
TABLE 1 | Primer information for PCR analysis.

\begin{tabular}{ll}
\hline Primer & Sequence $\left(\mathbf{5}^{\prime}\right.$ to $\mathbf{3}^{\prime} \mathbf{)}$ \\
\hline rol A-Forward & CATGTTCAGAATGGAATTA \\
rol A-Reverse & AGCCACGTGCGTATTAATCC \\
rol B-Forward & TCACAATGGATCCCAAATTG \\
rol B-Reverse & TCAAGTCGGCTTAGGCTT \\
rol C-Forward & ATGGCTGAAGACGACCTGTGT \\
rol C-Reverse & TAGCCGATTGCAAACTTGCA \\
rol D-Forward & ATGGCCAAACAACTTGCGA \\
rol D-Reverse & TAATGCCCGTGTCCATCG \\
\hline
\end{tabular}

using $1.2 \%$ gels that were stained with ethidium bromide and by measuring absorbance at 260:280 $\mathrm{nm}$ wavelength using a NanoVue Plus spectrophotometer. In total, $1 \mathrm{mg}$ of DNA-free total RNA was used to synthesize cDNA using transcriptase. The synthesized cDNA was further used for real time (RT)-PCR.

\section{Expression Patterns of Phenylpropanoid Biosynthetic Cluster Genes}

The BIO-RAD CFX96 Real-time PCR system (Bio-Rad Laboratories, Hercules, CA, USA) was used to determine the transcriptional levels of phenylpropanoid biosynthetic genes from the wild and transformed tartary buckwheat hairy roots. The primers for phenylpropanoid biosynthetic genes were designed as described by Li et al. (2010). Quantitative RT-PCR analysis was performed using gene-specific primer sets under the following conditions: initial denaturation at $95^{\circ} \mathrm{C}$ for $3 \mathrm{~min}$, followed by 40 cycles at $95^{\circ} \mathrm{C}$ for $15 \mathrm{~s}$, annealing at $55^{\circ} \mathrm{C}$ for $30 \mathrm{~s}$, and elongation at $72^{\circ} \mathrm{C}$ for $20 \mathrm{~s}$. The transcript levels of mRNA were compared relative to those of the standard histone $\mathrm{H} 3$ gene. The variations in expression levels were calculated by comparing three replicates of each sample.

\section{Extraction and Quantification of Phenolic Compounds Using HPLC}

To quantify the phenolic compounds in the transformed tartary buckwheat hairy roots, $100 \mathrm{mg}$ of the freeze-dried hairy root samples was powdered using a mortar and a pestle and was rinsed vigorously with $3 \mathrm{ml}$ of $100 \%$ methanol and, then, heated at $60^{\circ} \mathrm{C}$ for $1 \mathrm{~h}$ in a sonicator. The slurry was mixed intermittently every $20 \mathrm{~min}$ during the extraction. After $1 \mathrm{~h}$, the samples were centrifuged at 21,952 $\mathrm{rcf}$ for $10 \mathrm{~min}$, and the supernatant was filtered through a $0.45-\mathrm{mm}$ PTFE syringe filter for the HPLC analysis with a Futecs model NS-4000 HPLC apparatus (Daejeon, Korea). The operating conditions for HPLC and the separating protocols for individual phenolic compounds were according to the method of Li et al. (2010). Briefly, $20 \mu \mathrm{l}$ of the samples were injected; the HPLC column temperature was maintained at $30^{\circ} \mathrm{C}$; individual compounds were detected at $280 \mathrm{~nm}$. Mobile solutions were passed through the column at $1 \mathrm{ml} / \mathrm{min}$. The solutions consisted of a mixture of (A) MeOH:water:acetic acid (5:92.5:2.5, v/v/v) and (B) $\mathrm{MeOH}$ :water:acetic acid (95:2.5:2.5, v/v/v). The initial mobile phase composition was $0 \%$ solvent $\mathrm{B}$, followed by a linear gradient from 0 to $80 \%$ solvent $\mathrm{B}$ over $48 \mathrm{~min}$, and holding at $0 \%$ solvent B for an additional $10 \mathrm{~min}$. All phenolic compounds were calculated by comparing the HPLC peak areas with those of authentic standards, according to the procedures of Li et al. (2010).

\section{Quantification of Individual Anthocyanin Content Using HPLC}

For extraction of anthocyanins, $100 \mathrm{mg}$ of the powdered freezedried samples was mixed with $2 \mathrm{ml}$ of water:formic acid 95:5 $(\mathrm{v} / \mathrm{v})$ and maintained in the dark under shaking conditions. After proper mixing for $5 \mathrm{~min}$, the samples were incubated in a sonicator for $20 \mathrm{~min}$ with intermittent vortexing. After thorough shaking, the mixture was centrifuged at 11,200 rcf for $15 \mathrm{~min}$ at $4^{\circ} \mathrm{C}$. The collected debris-free supernatant was filtered and stored in a sterile brown bottle for HPLC analysis. Perkin Elmer Flexar HPLC equipped with a Security Guard Cartridges Kit AQ C18 column (4 mm; $63 \mathrm{~mm}$, i.d.) and a Synergi 4 m POLAR-RP 80A column (250 mm; $64.6 \mathrm{~mm}$, i.d., $4 \mathrm{~mm}$, particle size; Phenomenex, Torrance, CA, USA) was used for the separation and quantification of the individual anthocyanin content. The sample was eluted with a mixture of (A) water:formic acid (95:5, v/v) and (B) acetonitrile:formic acid $(95: 5, \mathrm{v} / \mathrm{v})$. The following gradient program was used: 0-8 $\mathrm{min}, 5-10 \%$ solvent $\mathrm{B} ; 8-13 \mathrm{~min}, 10-13 \%$ solvent $\mathrm{B}$; 13-15 min, $13 \%$ solvent $\mathrm{B}$; $15-18 \mathrm{~min}, 13-15 \%$ solvent $\mathrm{B}$; 18-25 min, $15 \%$ solvent $\mathrm{B} ; 5 \%$ solvent $\mathrm{B}$ at $25.1 \mathrm{~min}$; and, finally, 5\% solvent B for $10 \mathrm{~min}$ (total, $35 \mathrm{~min}$ ). Cyanidin-3$O$-glucoside and cyanidin-3-O-rutinoside standards were used for the quantification of individual anthocyanin content in the samples.

\section{Statistical Analysis}

A statistical analysis system (SAS version 9.2) was used for comparative analysis of gene expression of individual genes and phenolic components in the wild and transformed hairy roots of tartary buckwheat.

\section{RESULTS}

\section{Establishment of the Hairy Root Culture}

Wild-type A. rhizogenes (R1000) and strains R1200, R13333, R15834, R1601, LBA9402, and A4, that were isolated from crown gall disease plants, were used to determine the transformation efficiency. Mature seeds of tartary buckwheat were treated with different strains of $A$. rhizogenes to develop the hairy root culture. Primary results confirmed that the induction of hairy roots in tartary buckwheat is strain-specific because different $A$. rhizogenes strains exhibited varied infection efficiency patterns, hairy roots formation rates, and hairy root lengths. Among the A. rhizogenes strains R1000, R1200, LBA 9402, and A4, a $100 \%$ infection efficiency was observed. Whereas, the strain 15834 had comparatively lower efficiency (65\%; Table 2). Interestingly, each strain showed a similar infection type related 
TABLE 2 | Effects of different Agrobacterium rhizogenes strains on hairy root growth of Fagopyrum tataricum 'Hokkai T10'.

\begin{tabular}{lccr}
\hline $\begin{array}{l}\text { Agrobacterium } \\
\text { strain }\end{array}$ & $\begin{array}{c}\text { Infection } \\
\text { efficiency (\%) }\end{array}$ & $\begin{array}{l}\text { Number of hairy } \\
\text { roots/explants }\end{array}$ & $\begin{array}{c}\text { Length of } \\
\text { hairy root }(\mathbf{c m})\end{array}$ \\
\hline R 1000 & 100 & $5.3 \pm 0.6 a$ & $2.93 \pm 0.12 a$ \\
R1200 & 100 & $3.1 \pm 0.32 c$ & $2.27 \pm 0.23 b$ \\
13333 & 70 & $2.5 \pm 0.06 d$ & $2.2 \pm 0.35 b$ \\
15834 & 60 & $1.9 \pm 0.32 e$ & $1.53 \pm 0.06 c$ \\
R1601 & 65 & $2.1 \pm 0.06 e$ & $2.53 \pm 0.12 b$ \\
LBA 9402 & 100 & $3.8 \pm 0.06 b c$ & $2.33 \pm 0.15 b$ \\
A4 & 100 & $3.9 \pm 0.12 b$ & $2.43 \pm 0.06 b$ \\
\hline
\end{tabular}

to hairy roots and hairy root length. With respect to the hairy root length and the number of hairy roots, the strain R1000 (average, $2.93 \mathrm{~cm}$ and 5.3) was superior, followed by A4 and LBA 9402. Other strains showed a moderate number of hairy roots and slightly shorter hairy roots. However, no significant variations were observed among R1000, R1200, and 13333 strains. The occurrence of necrotic explant tissues in the form of hairy roots on a MS medium was fast and clearly indicated the transformation efficiency. Furthermore, the hairy root induction was comparatively faster (Figure 2). The dry weight of the developed hairy roots indicated that R1000 was dominant (0.4 g), followed by R3333 (0.41 g). Whereas, R1601 exhibited the lowest dry weight (Figure 3). Incubation period indicated the accumulation of phenolic compounds. PCR amplification of the related rol A, B, C, and D genes (300 bp, $750 \mathrm{bp}, 520 \mathrm{bp}$, and $1000 \mathrm{bp}$, respectively) in all of the transformed tartary buckwheat hairy roots confirmed the successful transformation.

\section{Expression of Phenylpropanoid Biosynthetic Pathway Genes}

To evaluate the expression levels of the genes related to the phenylpropanoid biosynthesis pathway in tartary buckwheat, the following genes were studied: ftpAL, FtC4H, Ft4CL, FrCHS, FrCH1, FrF3H, FtFLS1, FtFLS2, FtF3'H1, FtF3'H2, FtANS, and FtDFR. These genes encode PAL (phenylalanine ammonialyase), C4H (cinnamate 4-hydroxylase), 4CL (4-coumaroyl-CoA ligase), CHS (chalcone synthase), CHI (chalcone isomerase), $\mathrm{F} 3 \mathrm{H}$ (flavone 3-hydroxylase), FLS (flavonol synthase), F3' $\mathrm{H}$ (flavonoid 3'-hydroxylase), ANS (anthocyanidin synthase), and DFR (dihydroflavonol-4 reductase), respectively. Expression patterns of the selected genes in the transformed roots were strain-specific. All nine strains exhibited a wide range of gene expressions, which clearly indicated that each strain had a different gene regulation pattern in the tartary buckwheat host. In most cases, the transcript levels were comparatively higher for the strains 15834, A4, and R1000 (Figure 4). In general, the expression patterns of FtFLS1 and FtFLS2 were similar in all A. rhizogenes strains. R15834 showed a higher expression pattern compared with other strains. In contrast, R1000 did not enhance the expression levels of regulatory genes, such as $f t p A L$, FtC4H, Ft4CL, FtCHS, FtCHI, FtF3H, and FtF3'H-1. In roots that were infected with R1000, R1200, and 13333, the expression levels of FtFLS1, FtFLS2, FtF3'H2, and FtDFR were significantly higher relative to the transcript levels of the housekeeping gene (histone $\mathrm{H} 3$ ). These genes are directly involved in the biosynthesis of phenolic compounds in buckwheat. However, their relative expression in strains 15834, R1601, LBA9402, and A4 was lower compared with the other strains. In addition, LBA9402 and A4 showed significant expression for key enzymes related to the anthocyanin biosynthetic pathway (FtANS).

\section{Quantification of Phenolic Compounds in Hairy Roots of Tartary Buckwheat}

Individual content of phenolic compounds in hairy roots of tartary buckwheat is listed in Table 3. Six phenolic compounds were detected using HPLC. Irrespective of the strain, all six phenolic compounds were observed in the transformed hairy roots. Among the phenolic compounds, rutin $(7.14-23.31 \mu \mathrm{g} / \mathrm{mg}$ dry weight) showed the dominant amount in tartary buckwheat, whereas the content of rutin in the roots infected with R1000, R1200, 13333, and 15834 contributed an average of $92 \%$ of the total phenolic compounds. Infection with R1000 produced the highest rutin content $(22.31 \mu \mathrm{g} / \mathrm{mg}$ dry weight $)$. The observed levels of catechin, chlorogenic acid, ferulic acid, benzoic acid, and quercetin were less than $1 \mu \mathrm{g} / \mathrm{mg}$ dry weight.

\section{Quantification of Anthocyanins in Hairy Roots of Tartary Buckwheat}

Cyanidin derivatives of anthocyanins, such as cyanidin 3-Oglucoside and cyanidin 3-O-rutinoside, were detected in the hairy roots of tartary buckwheat using HPLC (Table 4). The results indicated that the amount of cyanidin 3-O-rutinoside (610-2400 $\mu \mathrm{g} / \mathrm{g}$ dry weight) was higher than that of cyanidin 3$O$-glucoside (300-800 $\mu \mathrm{g} / \mathrm{g}$ dry weight) in the infected tartary buckwheat roots. R1000-infected roots produced higher amounts of anthocyanins (3190 $\mu \mathrm{g} / \mathrm{g}$ dry weight), followed by R1200(2490), 13333- (2330), and 15834-infected roots (2280), whereas LBA 9402-infected roots showed the least total anthocyanin content (910). The average levels of cyanidin 3-O-glucoside and cyanidin 3-O-rutinoside in the treated samples were 400 and $1500 \mu \mathrm{g} / \mathrm{g}$ dry weight, respectively.

\section{DISCUSSION}

Plant metabolites and functional compounds have attracted interest because of their desired pharmaceutical properties and various useful applications in the medical field (Banerjee et al., 2012; Kaliyan and Agastian, 2015). Among plants, buckwheat is considered as a stable healthy food worldwide because of the presence of a wide variety of phytochemicals and nutrients, such as flavonoids, phenolic compounds, amino acids, and vitamins (Golisz et al., 2007; Alvarez-Jubete et al., 2010; Sedej et al., 2010). Buckwheat is a rich source of rutin (Hagels et al., 1995; Lachman et al., 2000). Rutin accumulates to the highest extent in buckwheat and cannot be found in other grains, such as wheat, rice, and corn. 


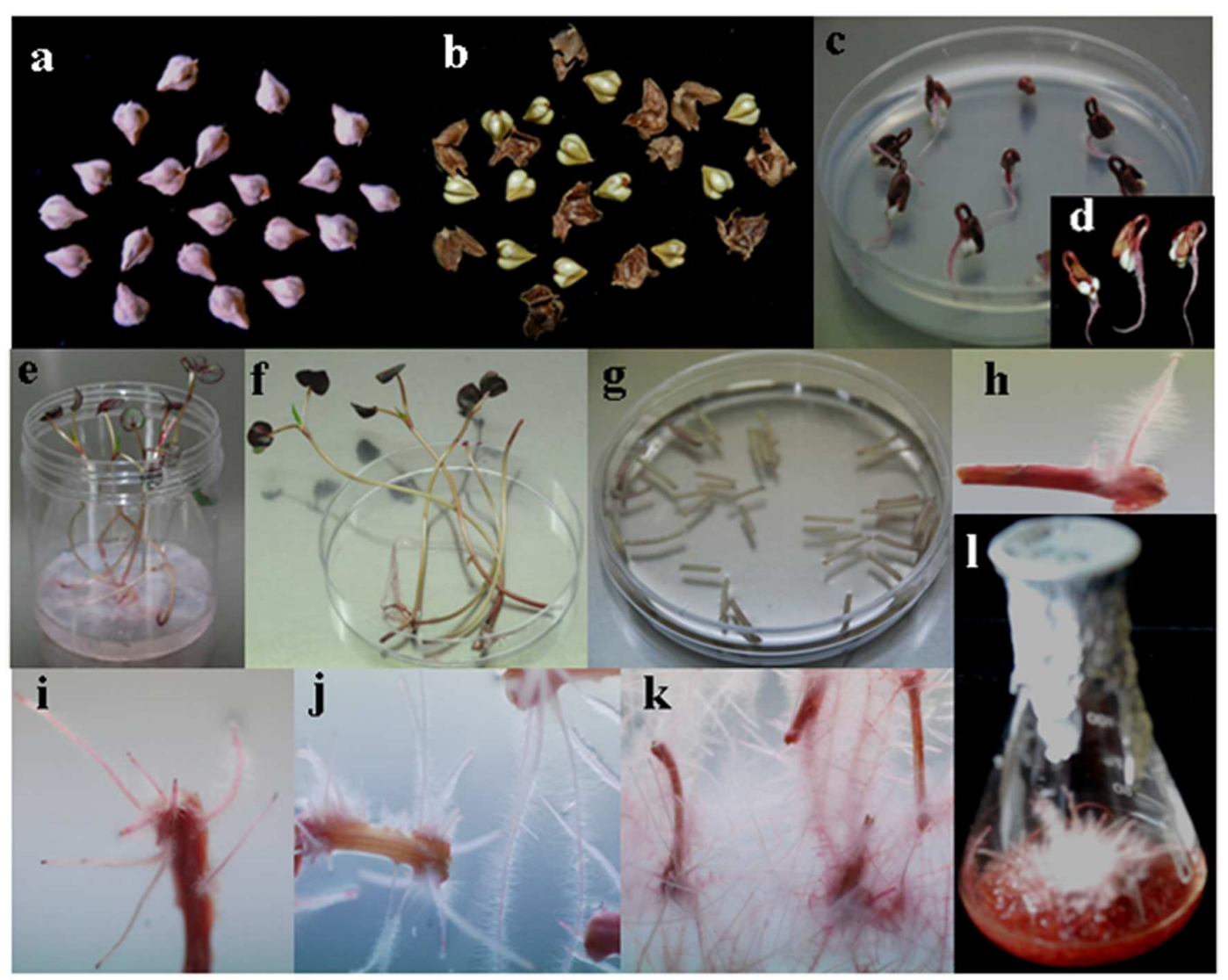

FIGURE 2 | Sequential stages of hairy root induction in Fagopyrum tataricum 'Hokkai T10'. (a) Hokkai T10 seeds. (b) De-hulled seeds. (c) Germinated seeds on a $1 / 2$ MS solid medium. (d) Three-day-old seedlings. (e) Five-day-old seedlings transferred to the Magenta box. (f) Explant preparation for Agrobacterium infection. (g) Explant infection in Agrobacterium inoculum. (h) Hairy root induction. (i) Profuse hairy roots 10 days after infection. (j) Hairy root growth 2 weeks after infection. (k) Rapid hairy root growth on a fresh medium. (I) Hairy root growth 14 days after culture in a 1/2 MS liquid medium.

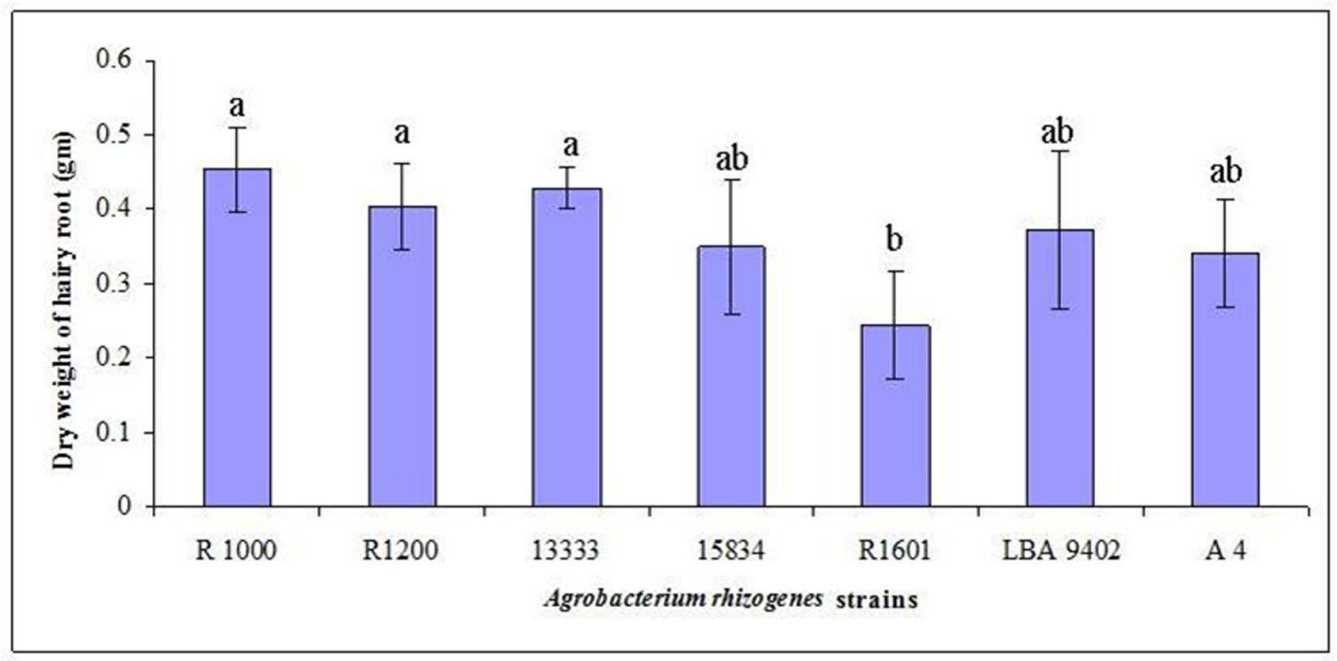

FIGURE 3 | Development of $\boldsymbol{F}$. tataricum 'Hokkai T10' hairy roots as influenced by different $\boldsymbol{A}$. rhizogenes strains. Data from three replicates were compared 14 days after culturing in a $1 / 2$ MS liquid medium. Mean values indicated by the same letter in a column are not different at $P<0.05$, according to Duncan's multiple range test. 


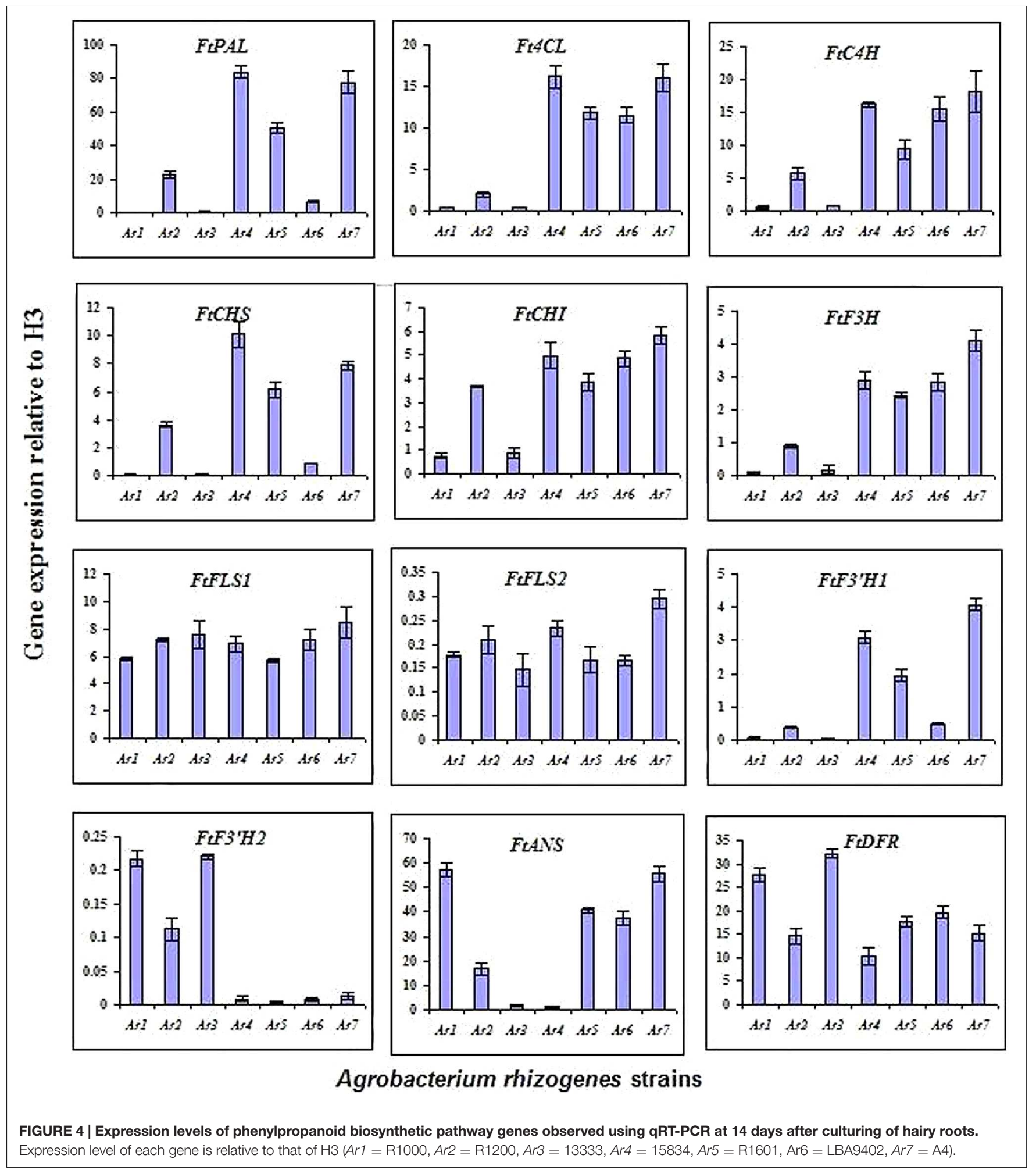

Thus, buckwheat is considered to be a major dietary source of rutin. Rutin has a wide range of pharmacological properties, such as anti-allergic, antioxidant, antimicrobial, antidiabetic, and anticancer properties (Al-Dhabi et al., 2015). Therefore, an accurate and reliable transformation method for the increased production of phenolic compounds, such as rutin and other phytochemicals in tartary buckwheat, is desired. A. tumefaciens and $A$. rhizogenes play an important role in the transformation of plant cell culture for the improved production of novel compounds. The development of hairy root culture using 
TABLE 3 | Content of phenolic compounds in hairy roots induced by different Agrobacterium rhizogenes strains ( $\mu \mathrm{g} / \mathrm{mg}$ dry weight).

\begin{tabular}{|c|c|c|c|c|c|c|}
\hline Agrobacterium strain & Catechin & Chlorogenic acid & Ferulic acid & Benzoic acid & Rutin & Quercetin \\
\hline R1000 & $0.53 \pm 0.02 a$ & $0.50 \pm 0.01 a$ & $0.30 \pm 0.01 c$ & $0.16 \pm 0.02 a$ & $22.31 \pm 0.54 a$ & $0.34 \pm 0.03 a$ \\
\hline $\mathrm{R} 1200$ & $0.53 \pm 0.01 a$ & $0.45 \pm 0.01 b$ & $0.27 \pm 0.01 c$ & $0.14 \pm 0.04 a$ & $21.96 \pm 0.65 a$ & $0.35 \pm 0.01 a$ \\
\hline 13333 & $0.36 \pm 0.06 b$ & $0.40 \pm 0.09 b c$ & $0.42 \pm 0.01 b$ & $0.10 \pm 0.01 b$ & $18.58 \pm 0.16 b$ & $0.34 \pm 0.01 a$ \\
\hline 15834 & $0.20 \pm 0.01 c$ & $0.34 \pm 0.02 c$ & $0.45 \pm 0.01 b$ & $0.09 \pm 0.01 b$ & $15.48 \pm 0.56 c$ & $0.30 \pm 0.02 a$ \\
\hline R1601 & $0.19 \pm 0.01 c$ & $0.43 \pm 0.03 b$ & $0.43 \pm 0.04 b$ & $0.07 \pm 0.01 b$ & $7.14 \pm 0.68 e$ & $0.39 \pm 0.01 a$ \\
\hline LBA 9402 & $0.19 \pm 0.01 c$ & $0.43 \pm 0.03 b$ & $0.43 \pm 0.04 b$ & $0.07 \pm 0.01 b$ & $7.14 \pm 0.68 e$ & $0.39 \pm 0.01 a$ \\
\hline A4 & $0.17 \pm 0.01 c$ & $0.47 \pm 0.01 a b$ & $0.76 \pm 0.01 a$ & $0.07 \pm 0.01 b$ & $13.04 \pm 0.01 c d$ & $0.32 \pm 0.02 a$ \\
\hline
\end{tabular}

TABLE 4 | Content of anthocyanin in hairy roots induced by different Agrobacterium rhizogenes strains ( $\mu \mathrm{g} / \mathrm{g}$ dry weight).

\begin{tabular}{lrrr}
\hline $\begin{array}{l}\text { Agrobacterium } \\
\text { strain }\end{array}$ & $\begin{array}{c}\text { Cyanidin } \\
\text { 3-O-glucoside }\end{array}$ & $\begin{array}{c}\text { Cyanidin } \\
\text { 3-O-rutinoside }\end{array}$ & \multicolumn{1}{l}{ Total } \\
\hline R 1000 & $800 \pm 0.00 \mathrm{a}$ & $2400 \pm 20.0 \mathrm{a}$ & $3190 \pm 20.0 \mathrm{a}$ \\
R1200 & $570 \pm 10.0 \mathrm{~d}$ & $1910 \pm 60.0 \mathrm{~b}$ & $2490 \pm 60.0 \mathrm{~b}$ \\
13333 & $490 \pm 0.00 \mathrm{e}$ & $1840 \pm 40.0 \mathrm{c}$ & $2330 \pm 40.0 \mathrm{c}$ \\
15834 & $750 \pm 10.0 \mathrm{~b}$ & $1530 \pm 30.0 \mathrm{~d}$ & $2280 \pm 30.0 \mathrm{c}$ \\
R1601 & $490 \pm 10.0 \mathrm{e}$ & $1080 \pm 20.0 \mathrm{f}$ & $1570 \pm 20.0 \mathrm{e}$ \\
LBA 9402 & $300 \pm 10.0 \mathrm{f}$ & $610 \pm 10.0 \mathrm{~g}$ & $910 \pm 20.0 \mathrm{f}$ \\
A 4 & $650 \pm 10.0 \mathrm{c}$ & $1170 \pm 10.0 \mathrm{e}$ & $1820 \pm 20.0 \mathrm{~d}$ \\
\hline
\end{tabular}

A. rhizogenes has various advantages over callus and cell culture due to gene stability and a relatively low cost and cultivation requirements (Banerjee et al., 2012). Moreover, A. rhizogenes, with its higher infection efficiency toward the plants, established new trends in the field of functional research for the production of major molecules that are similar to phenolic compounds. In the present study, the transformation efficiency of different $A$. rhizogenes strains, expression pattern of biosynthetic genes related to the production of phenolic compounds, and production capability of phenolic compounds were determined by inducing hairy roots in tartary buckwheat. The results indicated that all of the tested $A$. rhizogenes strains were able to induce hairy root formation with different transformation efficiencies. Zehra et al. (1999) studied the transformation efficiency of different $A$. rhizogenes strains for the production of novel metabolites in plants, and they confirmed that the hairy root induction is strain-specific. Generally, the $\mathrm{A}_{4}$ GUS strain was used for the hairy root induction in medicinal plants, such as Scutellaria baicalensis, Gentiana macrophylla, Hypericum perforatum, Aesculus hippocastanum, and Catharanthus roseus (Batra et al., 2004; Zdravković-Korać et al., 2004; Vinterhalter et al., 2006; Tiwari et al., 2007, 2008). Previously, we confirmed that rol gene insertions were observed in all of the strains and that the rol genes of the $\mathrm{Ri}$ plasmid in A. rhizogenes are responsible for the induction of hairy roots. However, the transformation efficiency of R1000 was superior to that of the other tested strains. Similarly, Tao and Li (2006) and Tiwari et al. (2007) reported that R1000 was efficient for developing a hairy root culture for medicinal plants.

Previously, we established a hairy root culture of F. tataricum and F. esculentum to produce phenolic compounds (Kim et al., 2009, 2010). To determine the gene transfer efficiency of different $A$. rhizogenes strains in the phenylpropanoid biosynthesis pathway in buckwheat, the expression levels of polypropanoid regulatory genes were studied. The results confirmed that, in most cases, the expression levels were comparatively higher for the R1000, A4, and 15834 strains. In addition, the expression levels for FtANS genes were directly related to anthocyanin biosynthesis in the transformed hairy roots. Similarly, Park et al. (2011) and Thwe et al. (2013) reported increased expression levels of FtANS genes in Hokkai T10' relative to in 'Hokkai T8' roots by $\mathrm{R} 1000$-mediated transformation. However, recently, Seo et al. (2015) claimed that the expression levels of FtDFR and FtFLS2 were similar. The increased transcript levels of FtDFR and FtANS were directly related to the content of leucoanthocyanidins and anthocyaninidins, which are precursors for the biosynthesis of cyanidin 3-O-glucoside and cyanidin 3-O-rutinoside, respectively. Our study confirmed that the expression levels of the regulatory genes for polypropanoid biosynthesis in buckwheat are directly related to the gene transfer efficiency of $A$. rhizogenes strains. Accumulation of the anthocyanin and phenolic compounds in hairy roots from the infection of different $A$. rhizogenes strains was not consistent with the expression of phenylpropanoid biosynthetic genes. We assumed that the expression level of phenylpropanoid biosynthetic genes in hairy roots from the infection of different $A$. rhizogenes strains would be different with respect to the source of the A. rhizogenes strains. However, we determined the expression of phenylpropanoid biosynthetic genes when we harvested the hairy roots.

Analysis of the individual phenolic compounds and anthocyanins contents in the transformed hairy root cultures showed the expression levels of the target genes in polypropanoid biosynthesis. Among the phenolic compounds, rutin and chlorogenic acid showed dominant levels in the hairy root culture. Previous studies have reported that the dry weight of tartary buckwheat seeds was $1.7 \%$ (Seo et al., 2015). Because rutin, which is present in tartary buckwheat, has various biological applications, its content can be increased by the hairy root culture. Similarly, the levels of anthocyanins, which have antioxidant, anti-inflammatory, antiviral, and antihistamine properties, could be enhanced in tartary buckwheat (RiceEvans and Packer, 2003). Trotin et al. (1993) and Lee et al. (2007) developed buckwheat hairy root cultures to enhance the production of phenolic compounds, such as rutin, chlorogenic 
acid, and caffeic acid. Overall, R1000 has been recommended as a promising strain for the hairy root induction and for the phenolic compound accumulation in buckwheat.

\section{CONCLUSION}

Gene transfer and hairy root stimulation in tartary buckwheat were affected by the $A$. rhizogenes strains used. Specifically, the genes that were involved in the metabolic pathway for the biosynthesis of polypropanoid were studied using nine $A$. rhizogenes strains. Transcript analysis clearly indicated that the expression of individual genes was strain-specific. However, mass production of hairy roots in buckwheat was efficiently established using the A. rhizogenes R1000 strain by following the protocol described in this study. Overall, the selection and identification of

\section{REFERENCES}

Al-Dhabi, N. A., Arasu, M. V., Park, C. H., and Park, S. U. (2015). An up-to-date review of rutin and its biological and pharmacological activities. EXCLI J. 14, 59-63. doi: 10.17179/excli2014-663

Ali, S. S., Kasoju, N., Luthra, A., Singh, A., Sharanabasava, H., Sahu, A., et al. (2008). Indian medicinal herbs as sources of antioxidants. Food Res. Int. 41, 1-15. doi: 10.1016/j.foodres.2007.10.001

Alvarez-Jubete, L., Wijngaard, H., Arendt, E. K., and Gallagher, E. (2010). Polyphenol composition and in vitro antioxidant activity of amaranth, quinoa buckwheat, and wheat as affected by sprouting and baking. Food Chem. 119, 770-778. doi: 10.1016/j.foodchem.2009.07.032

Banerjee, S., Singh, S., and Rahman, L. U. (2012). Biotransformation studies using hairy root cultures A review. Biotechnol. Adv. 30, 461-468. doi: 10.1016/j.biotechadv.2011.08.010

Batra, J., Ajaswrata, D., Singh, D., Kumar, S., and Sen, J. (2004). Growth and terpenoid indole alkaloid production in Catharanthus roseus hairy root clones in relation to left and right termini-linked Ri T-DNA gene integration. Plant Cell Rep. 23, 148-154. doi: 10.1007/s00299-004-0815-x

Berkov, S., Pavlov, A., Kovacheva, P., Stanimirova, P., and Philipov, S. (2003). Alkaloid spectrum in diploid and tetraploid hairy root cultures of Datura stramonium. Z. Naturforsch. C 58, 42-46.

Bonafaccia, G., Marocchini, M., and Kreft, I. (2003). Composition and technological properties of the flour and bran from common and tartary buckwheat. Food Chem. 80, 9-15. doi: 10.1016/S0308-8146(02)00228-5

Bourgaud, F., Gravot, A., Milesi, S., and Gontier, E. (2001). Production of plant secondary metabolites: a historical perspective. Plant Sci. 161, 839-851. doi: 10.1016/S0168-9452(01)00490-3

Bulgakov, V. P., Aminin, D. L., Shkryl, Y. N., Gorpenchenko, T. Y., Veremeichik, G. N., Dmitrenok, P. S., et al. (2008). Suppression of reactive oxygen species and enhanced stress tolerance in Rubia cordifolia cells expressing the rolC oncogene. Mol. Plant Microbe Interact. 21, 1561-1570. doi: 10.1094/MPMI-21-12-1561

Chan, P. K. (2003). Inhibition of tumor growth in vitro by the extract of Fagopyrum cymosum. Life Sci. 72, 1851-1858. doi: 10.1016/S0024-3205(03)00013-4

Georgiev, M. I., Agostini, E., Ludwig-Müller, J., and Xu, J. (2012). Genetically transformed roots: from plant disease to biotechnological resource. Trends Biotechnol. 30, 528-537. doi: 10.1016/j.tibtech.2012.07.001

Giri, A., and Narasu, M. L. (2000). Transgenic hairy roots: recent trends and applications. Biotechnol. Adv. 18, 1-22. doi: 10.1016/S0734-9750(99)00016-6

Golisz, A., Lata, S., and Gawronski, Y. F. (2007). Specific and total activities of the allele chemicals identified in buckwheat. Weed Biol. Manag. 7, 164-171. doi: 10.1111/j.1445-6664.2007.00252.x

Hagels, H., Wagenbreth, D., and Schilcher, H. (1995). Phenolic compounds of buckwheat herb and influence of plant and agricultural factors (Fagopyrum esculentum Moench. and Fagopyrum tataricum Gartner). Curr. Adv. Buckwheat Res. 115, 801-809. a suitable $A$. rhizogenes strain is useful for improving the production of phenolic compounds and anthocyanins in buckwheat.

\section{AUTHOR CONTRIBUTIONS}

All authors listed, have made substantial, direct and intellectual contribution to the work, and approved it for publication.

\section{ACKNOWLEDGMENT}

The authors extend their sincere appreciation to the Deanship of Scientific Research at King Saud University for its funding this Prolific Research Group (PRG-1437-28).

Ishii, S., Katsumura, T., Shiozuka, C., Ooyauchi, K., Kawasaki, K., Takigawa, S., et al. (2008). Anti-inflammatory effect of buckwheat sprouts in lipopolysaccharide-activated human colon cancer cells and mice. Biosci. Biotechnol. Biochem. 72, 3148-3157. doi: 10.1271/bbb. 80324

Kaliyan, B. K., and Agastian, P. (2015). In vitro regeneration of a rare antidiabetic plant Epaltes divaricata L. South Indian J. Biol. Sci. 1, 52-59.

Kawa, J. M., Taylor, C. G., and Przybylski, R. (2003). Buckwheat concentrate reduces serum glucose in streptozotocin diabetic rats. J. Agric. Food Chem. 51, 7287-7291. doi: 10.1021/jf0302153

Kayashita, J., Shimaoka, I., Nakajoh, M., Yamazaki, M., and Norihisa, K. (1997). Consumption of buckwheat protein lowers plasma cholesterol and raises fecal neutral sterols in cholesterol-fed rats because of its low digestibility. J. Nutr. 127, 1395-1400.

Kim, Y. K., Li, X., Xu, H., Park, N., Uddin, M. R., Pyon, J. Y., et al. (2009). Production of phenolic compounds in hairy root culture of tartary buckwheat (Fagopyrum tataricum Gaertn). J. Crop Sci. Biotechnol. 12, 53-57. doi: 10.1371/journal.pone.0065349

Kim, Y. K., Xu, H., Park, W. T., Park, N. I, Lee, S. Y., and Park, S. U. (2010). Genetic transformaiton of buckwheat (Fagopyrum esculentum M.) with Agrobacterium rhizogenes and production of ruin in transformed root cultures. Aust. J. Crop Sci. 4, 485-490.

Kwon, B. M., Ro, S. H., Kim, M. K., Nam, J. Y., Jung, H. J., Lee, I. R., et al. (1997). Polyacetilene analogs isolated from hairy roots of Panax ginseng, inhibit acyl-CoA-cholesterol. Planta Med. 63, 552-553. doi: 10.1055/s-2006-9 57763

Lachman, J., Orsak, M., Pivec, V., and Faustusova, E. (2000). Content of rutin in selected plant sources. Sci. Agric. 31, 89-99.

Lee, S.-W., Seo, J. M., Lee, M.-K., Chun, J.-H., Antonisamy, P., Arasu, M. V., et al. (2014). Influence of different LED lamps on the production of phenolic compounds in common and Tartary buckwheat sprouts. Ind. Crops Prod. 54, 320-326. doi: 10.1016/j.indcrop.2014.01.024

Lee, S. Y., Cho, S. I., Park, M. H., Kim, Y. K., Choi, J. E., and Park, S. U. (2007). Growth and rutin production in hairy root cultures of buckwheat (Fagopyrum esculentum M.). Prep. Biochem. Biotechnol. 37, 239-246. doi: 10.1080/10826060701386729

Li, W., Asada, Y., and Yoshikawa, T. (1998). Antimicrobial flavonoids from Glycyrrhiza glabra hairy root cultures. Planta Med. 64, 746-747. doi: 10.1055/s2006-957571

Li, X., Park, N. I., Woo, S. H., Park, C. H., and Park, S. U. (2010). Differential expression of flavonoid biosynthesis genes and accumulation of phenolic compounds in common buckwheat (Fagopyrum esculentum). J. Agric. Food Chem. 58, 12176-12181. doi: 10.1021/jf103310g

Murashige, T., and Skoog, F. (1962). A revised medium for rapid growth and bioassays with tobacco tissue cultures. Physiol. Plant. 15, 473-497. doi: 10.1111/j.1399-3054.1962.tb08052.x 
Nagella, P., Thiruvengadam, M., Jung, S. J., Murthy, H. N., and Chung, I. M. (2013). Establishment of Gymnema sylvestre hairy root cultures for the production of gymnemic acid. Acta Physiol. Plant. 35, 3067-3073. doi: 10.1007/s11738-0131327-5

Ooi, C. T., Syahida, A., Stanslas, J., and Maziah, M. (2013). Efficiency of different Agrobacterium rhizogenes strains on hairy roots induction in Solanum mammosum. World J. Microbiol. Biotechnol. 29, 421-430. doi: 10.1007/s11274012-1194-Z

Park, N. I., Li, X., Suzuki, T., Kim, S. J., Woo, S. H., Park, C. H., et al. (2011). Differential expression of anthocyanin biosyntheticgenes and anthocyanin accumulation in tartary buckwheat cultivars 'Hokkai T8' and 'Hokkai T10'. J. Agric. Food Chem. 59, 2356-2361. doi: 10.1021/jf200020b

Park, S. U., and Facchini, P. J. (2000). Agrobacterium rhizogenes-mediated stable transformation of opium poppy, Papaver somniferum and California poppy, Eschscholzia californica Cham., root cultures. J. Exp. Bot. 51, 1005-1016. doi: 10.1093/jexbot/51.347.1005

Rice-Evans, C. A., and Packer, L. (2003). Flavonoids in Health and Disease. New York, NY: Marcel Dekker Inc, 467-469.

Sedej, I., Mandić, A., Sakač, M., Mišan, A., and Tumbas, V. (2010). Comparison of antioxidant components and activity of buckwheat and wheat flours. Cereal Chem. J. 87, 387-392. doi: 10.1094/CCHEM-02-10-0018

Seo, J.-M., Arasu, M. V., Kim, Y.-B., Park, S. U., and Kim, S.-J. (2015). Phenylalanine and LED lights enhance phenolic compound production in Tartary buckwheat sprouts. Food Chem. 177, 204-213. doi: 10.1016/j.foodchem.2014.12.094

Shanks, J. V., and Morgan, J. (1999). Plant "hairy root" culture. Curr. Opin. Biotechnol. 10, 151-155. doi: 10.1016/S0958-1669(99)80026-3

Sharma, P., Padh, H., and Shrivastava, N. (2013). Hairy root cultures: a suitable biological system for studying secondary metabolic pathways in plants. Eng. Life Sci. 13, 62-75. doi: 10.1002/elsc.201200030

Shen, W. H., Petit, A., Guern, J., and Tempe, J. (1988). Hairy roots are more sensitive to auxin than normal roots. Proc. Natl. Acad. Sci. U.S.A. 35, 3417-3421. doi: 10.1073/pnas.85.10.3417

Sudha, C. G., Sherina, T. V., Anu Anand, V. P., Reji, J. V., Padmesh, P., and Soniya, E. V. (2013). Agrobacterium rhizogenes mediated transformation of the medicinal plant Decalepis arayalpathra and production of 2-hydroxy4-methoxy benzaldehyde. Plant Cell Tissue Organ Cult. 112, 217-226. doi: 10.1007/s11240-012-0226-6

Tao, J., and Li, L. (2006). Genetic transformation of Torenia fournieri L. mediated by Agrobacterium rhizogenes. S. Afr. J. Bot. 72, 211-216. doi: 10.1016/j.sajb.2005.07.010

Thwe, A. A., Kim, J. K., Li, X., Kim, Y. B., Uddin, M. R., Kim, S. J., et al. (2013). Metabolomic analysis and phenylpropanoid biosynthesis in hairy root culture of tartary buckwheat cultivars. PLoS ONE 8:e65349. doi: 10.1371/journal.pone.0065349

Tiwari, R. K., Trivedi, M., Guang, Z.-C., Guo, G.-Q., and Zheng, G.-C. (2007). Genetic transformation of Gentiana macrophylla with Agrobacterium rhizogenes: growth and production of secoiridoid glucoside gentiopicroside in transformed hairy root cultures. Plant Cell Rep. 26, 199-210. doi: 10.1007/s00299-006-0236-0

Tiwari, R. K., Trivedi, M., Guang, Z.-C., Guo, G.-Q., and Zheng, G.-C. (2008). Agrobacterium rhizogenes mediated transformation of Scutellaria baicalensis and production of flavonoids in hairy roots. Biol. Plant. 52, 26-35. doi: 10.1007/s10535-008-0004-9

Trotin, F., Moumou, Y., and Vasseur, J. (1993). Flavanol production by Fagopyrum esculentum hairy and normal root cultures. Phytochemistry 32, 929-931. doi: 10.1016/0031-9422(93)85231-F

Tzfira, T., Vaidya, M., and Citovsky, V. (2004). Involvement of targeted proteolysis in plant genetic transformation by Agrobacterium. Nature 431, 87-92. doi: 10.1038 /nature02857

Vinterhalter, B., Ninkovic, S., Cingel, A., and Vinterhalter, D. (2006). Shoot and root culture of Hypericum perforatum L. transformed with Agrobacterium rhizogenes A4M70GUS. Biol. Plant. 50, 767-770. doi: 10.1007/s10535-0060127-9

Wysokinska, H., and Chmiel, A. (1997). Transformed root cultures for biotechnology. Acta Biotechnol. 17, 131-159. doi: 10.1002/abio.370170205

Zdravković-Korać, S., Muhovski, Y., Druart, P., Calic, D., and Radojevic, L. (2004). Agrobacterium rhizogenes mediated DNA transfer to Aesculus hippocastaneum L. and the regeneration of transformed plants. Plant Cell Rep. 22, 698-704. doi: 10.1007/s00299-004-0756-4

Zehra, M., Banerjee, S., Sharma, S., and Kumar, S. (1999). Influence of Agrobacterium rhizogenes strains on biomass and alkaloid productivity in hairy root lines of Hyoscyamus muticus and H. albus. Planta Med. 64, 60-63. doi: $10.1055 /$ s-1999-13964

Conflict of Interest Statement: The authors declare that the research was conducted in the absence of any commercial or financial relationships that could be construed as a potential conflict of interest.

Copyright (c) 2016 Thwe, Valan Arasu, Li, Park, Kim, Al-Dhabi and Park. This is an open-access article distributed under the terms of the Creative Commons Attribution License (CC BY). The use, distribution or reproduction in other forums is permitted, provided the original author(s) or licensor are credited and that the original publication in this journal is cited, in accordance with accepted academic practice. No use, distribution or reproduction is permitted which does not comply with these terms. 